\title{
Estimation of the Parameters for the Exponentiated Weibull Distribution Based on Progressive Type-II Censoring Scheme
}

\author{
Mohammed Mohammed Ahmed Almazah \\ Correspondence: Mathematics Department, College of Sciences and Arts (Mayhill), King Khalid University., KSA; \\ Mathematics and Computer Department, College of sciences, Ibb University., Yemen
}

Received: June 8, 2020 Accepted: June 29, 2020 Online Published: July 28, 2020

doi:10.5539/ijsp.v9n5p1 URL: https://doi.org/10.5539/ijsp.v9n5p1

\begin{abstract}
The main objective of the present study is to find the estimation of the two Exponentiated Weibull distribution parameters, based on progressive Type II censored samples. The maximum likelihood and Bayes estimators for the two shape parameters and the scale parameter of the exponentiated Weibull lifetime model were derived. Bayes estimators was obtained by using both the symmetric and asymmetric loss functions via squared error loss and linex loss functions This was done with respect to the conjugate priors for two shape parameters. We used an approximation based on the Lindley (Trabajos de Estadistca) method for obtaining Bayes estimates under these loss functions. The different proposed estimators have been compared through an extensive simulation studies. Bayes ratings also turned out to be better than MLE. Whatever the sample sizes are, we get the same results.
\end{abstract}

Keywords: bayes estimation, LINEX loss function, maximum likelihood estimates, exponentiated weibull model

\section{Introduction}

The Exponentiated Weibull distribution is considered an important statistical distribution. The importance of this distribution lies in the fact that the function of the survival rate takes different forms, which make it suitable for studying and covering many problems of reconciliation of the times of life and validity. It contains many probability distributions as special cases.

The form of the probability density function (PDF) and cumulative distribution function (CDF), with two shape parameters, $\alpha$ and $\theta$ are given, respectively, by

$$
f(t)=\alpha \theta t^{\alpha-1} e^{-t^{\alpha}}\left(1-e^{-t^{\alpha}}\right)^{\theta-1} \quad t>0, \alpha>0, \theta>0
$$

where $\alpha, \theta$ : shape parameter and

$$
F(t)=\left(1-e^{-t^{\alpha}}\right)^{\theta} \quad, t>0
$$

Also, the Reliability function of the Exponentiated Weibull distribution with two shape parameters $\alpha$ and $\theta$ is in the formula

$$
\mathrm{R}(\mathrm{t})=1-\left(1-\mathrm{e}^{-\mathrm{t}^{\alpha}}\right)^{\theta} \quad, \mathrm{t}>0
$$

Failure function is written in the formula:

$$
\mathrm{h}(\mathrm{t})=\alpha \theta \mathrm{t}^{\alpha-1} \mathrm{e}^{-\mathrm{t}^{\alpha}}\left(1-\mathrm{e}^{-\mathrm{t}^{\alpha}}\right)^{\theta-1}\left[1-\left(\left(1-\mathrm{e}^{-\mathrm{t}^{\alpha}}\right)^{\theta-1}\right)\right]
$$

The maximum likelihood estimations of the distribution are discussed by Mudholkar et al. (1995) using several sets of data. If the shape parameter $\theta=1$ the EW probability density function (PDF) is that of the Weibull distribution. Other statistical properties of this distribution are discussed by Mudholkar and Hutson (1996) and Nassar and Eissa (2003). Parametric characterizations of the density function are discussed by Jiang and Murthy (1999) and Soliman (2000, 2001). They showed that the density function of the EW distribution is decreasing when $\alpha \theta \leq 1$ and unimodal when $\alpha \theta>$ 1. Some statistical properties of this distribution are discussed by Jiang and Murthy (1999), and Nassar and Eissa (2004) that derived Bayes estimates of the two shape parameters, reliability and failure rate functions of the exponential Weibull lifetime model, from complete and Type II censored and complete samples. 
Choudhury (2005) proposed a simple derivation for the moments of the EW. Singh et al. (2005a,b) derived maximum likelihood and Bayes estimates for the parameters of the EWM based on Type-II censored samples. (Pal et al, 2006) introduced many properties and obtained some inferences for the three parameters EW. Kim et al. (2011) obtained the maximum likelihood and Bayes estimators for the two shape parameters and the reliability function of the EW based on progressive Type-II censored samples.

In many life test studies, it is common that the lifetimes of test units may not be able to record exactly. For example, in Type II censoring, the test terminates after a predetermined number of failures occurs in order to save time or cost. Furthermore, some test units may have to be removed at different stages in the study for various reasons. This would lead to progressive censoring. Although censoring occurs frequently in many applications, there are relatively few research projects that have published in this specific area. Some early studies can be found in Cohen (1963); Confild(1970); Mann (1971); Zellner(1986); Pandey and Rai(1992); Viveros and Balakrishnan (1994)) and Balakrishnan and Sandhu (1995)and Pandey(1996).

\section{Method}

Suppose that $\mathrm{n}$ independent items are put on a life test and the censoring scheme $\left(\mathrm{R}_{1}, \mathrm{R}_{2}, \cdots, \mathrm{R}_{\mathrm{n}}\right)$ is previously fixed such that immediately following the first failure, $t_{1}, R_{1}$ surviving items are removed from the experiment at random and immediately following the second failure, $t_{2}, R_{2}$ surviving items are removed from the experiment at random; this process continues until, at the time of the mth observed failure, $\mathrm{tm}$, the remaining $\mathrm{Rm}$ items are removed from the test. The $\mathrm{n}$ ordered observed failure times denoted byt $_{1}, \ldots, \mathrm{t}_{\mathrm{n}}$ are called progressive Type II right censored order statistics of size $m$ from a sample of size $n$ with a progressive censoring scheme $\left(R_{1}, R_{2}, \cdots, R_{n}\right)$. It is clear that $m=$ $n+R_{1}+R_{2}+\cdots+R_{n}$. Also that the familiar complete and Type II right-censored samples are special cases of this scheme. If $\mathrm{R} 1=\mathrm{R} 2=\cdots=\mathrm{Rn}-1=0$ and $\mathrm{Rn}=\mathrm{m}-\mathrm{n}$, this sampling scheme reduces to conventional Type II censoring. Also, if $\mathrm{R} 1=\mathrm{R} 2=\cdots=\mathrm{Rn}=0$, then the progressive Type II censoring scheme reduced to the complete sampling case. If the failure times of the items originally on the test with progressive censoring scheme (R1, R2, . , Rn) are from a continuous population with probability density function $f(x)$ and cumulative density function $F(t)$, then the joint probability density function of progressively Type II censored sample is:

$\underline{X}=X_{1, m, n}^{\left(R_{1}, R_{2}, \ldots, R_{m}\right)}, X_{2, m, n}^{\left(R_{1}, R_{2}, \ldots, R_{m}\right)}, \ldots, X_{m, m, n}^{\left(R_{1}, R_{2}, \ldots, R_{m}\right)}$ of size $m$ form a sample of size $n$ is given by:

$$
\begin{gathered}
f_{\mathrm{t}(1, \mathrm{n}, \mathrm{m} \mathrm{k}) \ldots . ., \mathrm{t}(\mathrm{n}, \mathrm{n}, \mathrm{m}, \mathrm{k})}\left(\mathrm{t}_{1}, \mathrm{t}_{2}, \ldots ., \mathrm{t}_{\mathrm{n}}\right)=\mathrm{d}_{\mathrm{r}-1}\left[\prod_{\mathrm{i}=1}^{\mathrm{r}-1}\left[\overline{\mathrm{F}}\left(\mathrm{t}_{\mathrm{i}}\right)\right]^{\mathrm{m}_{1}} \mathrm{f}\left(\mathrm{t}_{\mathrm{i}}\right)\right]\left[\overline{\mathrm{F}}\left(\mathrm{t}_{\mathrm{n}}\right)\right]^{\mathrm{k}-1} \mathrm{f}\left(\mathrm{t}_{\mathrm{n}}\right), \\
\mathrm{F}^{-1}(0)<\mathrm{t}_{(1)} \leq \mathrm{t}_{(2)} \leq \cdots \leq \mathrm{t}_{(\mathrm{n})}<\mathrm{F}^{-1}(1),
\end{gathered}
$$

Where

$$
\mathrm{d}_{\mathrm{n}-1}=\mathrm{k}\left(\prod_{\mathrm{i}=1}^{\mathrm{n}-1} \gamma_{\mathrm{i}}\right) \quad, \quad \gamma_{\mathrm{i}}=\mathrm{k}+\mathrm{n}-\mathrm{j}+\prod_{i=j}^{n-1} m_{1}>0
$$

$$
\text { For } \mathrm{j} \in\{1,2, \ldots, \mathrm{n}\} \text { and } \overline{\mathrm{F}}(\mathrm{t})=1-\mathrm{F}(\mathrm{t})
$$

\subsection{Maximum Likelihood Estimation}

Suppose that the lifetimes of the unite being tested have a Weibull distribution. The probability density function is given in Equation (1). Let $t=\left(t_{1}, t_{2}, \ldots, t_{n}\right)$ be a progressively type II conserved sample a Weibull distribution, with censoring scheme $r=\left(r_{1}, r_{2}, \ldots, r_{n}\right)$.Based on the observed data (t.r), the likelihood function can be obtained substituting from(1) and (2) in (5)written as:

where

$$
\begin{gathered}
\ell(\alpha, \theta \mid t)=d_{m-1} \alpha^{m} \theta^{m} \prod_{j=1}^{m} \psi_{j} \eta_{j}^{\theta} \prod_{j=1}^{m-1} \eta_{j}^{n \theta} \eta_{n}^{\theta(k-1)} \\
t=\left(t_{1}, t_{2}, \ldots, t_{m}\right) \text { for } j=1,2, \ldots, n \\
\psi_{j}=\frac{t_{j}^{\alpha-1} e^{-t_{j}^{\alpha}}}{\eta_{j}} \\
\eta_{j}=1-e^{-t_{j}^{\alpha}}
\end{gathered}
$$

The natural logarithm of the likelihood function (1) is given by:

$$
\mathrm{L}(\alpha, \theta \mid \mathrm{t})=\ln \left(\mathrm{d}_{\mathrm{m}-1}\right)+\mathrm{m} \ln \alpha+\mathrm{m} \ln \theta+\sum_{\mathrm{j}=1}^{\mathrm{m}}\left(\ln \psi_{\mathrm{j}}+\theta \ln \eta_{\mathrm{j}}\right)+\mathrm{n} \theta \sum_{\mathrm{j}=1}^{\mathrm{m}-1} \ln \eta_{\mathrm{j}}+\theta(\mathrm{k}-1) \ln \eta_{\mathrm{n}}
$$


Taking derivatives with respect to $\alpha$ and $\theta$ and equating them zero, the obtained the likelihood equations for $\alpha$ and $\theta$

$$
\frac{\partial \mathrm{L}(\alpha, \theta \mid \mathrm{t})}{\partial \alpha}=\frac{\mathrm{m}}{\alpha}+\sum_{\mathrm{j}=1}^{\mathrm{m}}\left(\xi_{1 \mathrm{j}}(\alpha)+(\theta-1) \xi_{2 \mathrm{j}}(\alpha)\right)+\mathrm{n} \theta \sum_{\mathrm{j}=1}^{\mathrm{m}-1} \xi_{2 \mathrm{j}}(\alpha)+\theta(\mathrm{k}-1) \xi_{2 \mathrm{j}}(\alpha)
$$

and

$$
\frac{\partial \mathrm{L}(\alpha, \theta \mid \mathrm{t})}{\partial \theta}=\frac{\mathrm{m}}{\theta}+\sum_{\mathrm{j}=1}^{\mathrm{m}} \ln \eta_{\mathrm{j}}+\mathrm{n} \sum_{\mathrm{j}=1}^{\mathrm{m}-1} \ln \eta_{\mathrm{j}}+(\mathrm{k}-1) \ln \eta_{\mathrm{n}}=0
$$

where

$$
\begin{gathered}
\xi_{1 j}(\alpha)=\left(1-t_{j}^{\alpha}\right) \ln t_{j}, \\
\xi_{2 j}(\alpha)=\frac{t_{j}^{\alpha} e^{-t^{\alpha}} \ln t_{j}}{\eta_{j}} \\
\text { For } j=1,2, \ldots, n
\end{gathered}
$$

Obtaining the closed forms equation (8) and (9) are not possible. The solution can be obtained by using the Newton-Raphson method. For a given $t$, the Maximum Likelihood Estimation (MLE) of the reliability function $R(t)$ is computed form(3) after replacing $\alpha$ and $\theta$ by their MLE estimation.

The asymptotic variance-covariance matrix of the MLE for parameters $\alpha$ and $\theta$ is given by the elements of the Fisher information matrix

$$
I_{i j}=E\left(\frac{-\partial^{2} L(\alpha, \theta \mid t)}{\partial \alpha \partial \theta}\right), i, j=1,2
$$

But, the exact mathematical expressions for the above expressions are very difficult to obtain. Therefore, we give the approximate asymptotic variance-covariance matrix for the MLE. Which is obtained by dropping the expression operators as following:

$$
\left[\begin{array}{cc}
\operatorname{var}(\widehat{\alpha}) & \operatorname{cov}(\widehat{\alpha}, \hat{\theta}) \\
\operatorname{cov}(\widehat{\alpha}, \hat{\theta}) & \operatorname{var}(\hat{\theta})
\end{array}\right]=\left[\begin{array}{cc}
\frac{-\partial^{2} \mathrm{~L}(\alpha, \theta)}{\partial \alpha^{2}} & \frac{-\partial^{2} \mathrm{~L}(\alpha, \theta)}{\partial \alpha \partial \theta} \\
\frac{-\partial^{2} \mathrm{~L}(\alpha, \theta)}{\partial \alpha \partial \theta} & \frac{-\partial^{2} \mathrm{~L}(\alpha, \theta)}{\partial \theta^{2}}
\end{array}\right]
$$

With:

$$
\begin{gathered}
\frac{\partial^{2} \mathrm{~L}}{\partial \theta^{2}}=\frac{-\mathrm{m}}{\theta^{2}} \\
\frac{\partial^{2} \mathrm{~L}}{\partial \alpha^{2}}=\frac{-\mathrm{m}}{\alpha^{2}}-\sum_{\mathrm{j}=1}^{\mathrm{m}} \mathrm{t}_{\mathrm{j}}^{\alpha} \ln ^{2} \mathrm{t}_{\mathrm{j}}+\mathrm{n} \theta \sum_{\mathrm{j}=1}^{\mathrm{m}-1} \gamma_{\mathrm{j}}+\sum_{\mathrm{j}=1}^{\mathrm{m}}(\theta-1) \gamma_{\mathrm{j}}+\theta(\mathrm{k}-1) \gamma_{\mathrm{n}} \\
\frac{\partial^{2} \mathrm{~L}}{\partial \alpha \partial \theta}=\frac{\partial^{2} \mathrm{~L}}{\partial \theta \partial \alpha}=\sum_{\mathrm{j}=1}^{\mathrm{m}} \xi_{2 \mathrm{j}}(\alpha)+\mathrm{n} \sum_{\mathrm{j}=1}^{\mathrm{m}-1} \xi_{2 \mathrm{j}}(\alpha)+(\mathrm{k}-1) \xi_{2 \mathrm{j}}(\alpha)
\end{gathered}
$$

where

$$
\begin{gathered}
\gamma_{\mathrm{j}}=\theta \xi_{2 \mathrm{j}}(\alpha)\left[\xi_{1 \mathrm{j}}(\alpha)-\xi_{2 \mathrm{j}}(\alpha)\right] \\
\text { For } \mathrm{j}=1,2, \ldots, \mathrm{n}
\end{gathered}
$$

The asymptotic normality of the MLE can be used to compute the approximate confidence intervals for parameters $\alpha$ and $\theta$. Therefore, $(1-\alpha) 100 \%$ confidence intervals for parameters $\alpha$ and $\theta$ have become respectively.

where $Z_{\frac{\alpha}{2}}$ : is a standard normal variant.

$$
\widehat{\alpha}_{M L}+Z_{\frac{\alpha}{2}} \sqrt{\operatorname{var}\left(\widehat{\alpha}_{M L}\right)} \text { and } \hat{\theta}_{M L}+Z_{\frac{\alpha}{2}} \sqrt{\operatorname{var}\left(\hat{\theta}_{M L}\right)}
$$




\subsection{Bayes Estimation}

The Bayesian inference procedures have been developed under the usual SE loss function (quadratic loss), which is symmetrical, and associates equal importance to the losses due to overestimation and underestimation of equal magnitude. However, such a restriction may be impractical. For example, in the estimation of reliability and failure rate functions, an overestimation is usually more serious than an underestimation; the use of symmetric loss functions may be inappropriate, as has been recognized by Calabria and Pulcini (1996), Singh et al. (2002) and Singh, et al (2005a, b).

A very useful asymmetric loss function known as the LINEX loss function was first introduced by (Varian, 1975) and was widely used by several authors. This function rises approximately exponentially on one side of zero and approximately linearly on the other side. Under the assumption that the minimal loss occurs at $\hat{\lambda}=\lambda$, the LINEX loss function for $\lambda=\lambda(\alpha, \theta)$ can be expressed as:

$$
\mathrm{L}(\Delta) \propto\left(\mathrm{e}^{\mathrm{k} \Delta}-\mathrm{k} \Delta-1\right) \quad ; \mathrm{k} \neq 1
$$

where $\Delta=\hat{\lambda}-\lambda, \mathrm{k}$ is a constant amount that represents the shape parameter of the $\mathrm{L}(\Delta)$ function, $\mathrm{k}$ Fixed amount represents the shape parameter of the $\mathrm{L}$ function, which is of the most important properties:

1. It is asymmetric around the origin point.

2. The shape parameter controls the degree and direction of the asymmetry of the function, since the value of the numerical $\mathrm{k}$ parameter controls the degree of asymmetry of the loss function.

3 . The signal of parameter $\mathrm{k}$ reflects the direction of asymmetry, and degree of symmetry, respectively, $(\mathrm{k}>0)$ means overestimation is more serious than underestimation, and $\mathrm{k}<0$ ) means the opposite). For a closed to zero, the LINEX loss function is approximately the squared error (SE) loss, and therefore almost symmetric. Many researchers have used the linear loss function in it is various forms to obtain Bayesian inferences of the various statistical distributions. The Balanced Loss Function (BLF) as presented by (Zellner, 1996) is written in the formula:

$$
\mathrm{L}_{\rho, \lambda, \xi}^{\mathrm{q}}(\gamma(\theta), \delta)=\lambda \mathrm{q}(\theta) \rho(\mathrm{r}(\theta), \delta)+(1-\lambda) \mathrm{q}(\theta) \rho(\mathrm{r}(\theta), \delta)
$$

where $\delta$ is the parameter estimate $\gamma(\theta), \xi$ is a prior estimate of the parameter $\gamma(\theta), \lambda \in[0,1)$ and $\rho(\operatorname{r}(\theta), \delta)$ : is an optional loss function to estimate $\gamma(\theta)$ by $\delta$. In this study, the balanced squared error loss function and balanced LINEX loss function will be used by Ahmadi et al. (2009a,b), in the form

$$
\mathrm{L}_{\omega, \xi}(\gamma(\theta), \delta)=\lambda(\delta-\xi)^{2}+(1-\lambda) \rho(\delta-\gamma(\theta))^{2}
$$

and the corresponding Bayes estimate of the function $\gamma(\theta)$ is given by

$$
\delta_{\varpi, \xi}(\underline{\mathrm{t}})=\vartheta \delta+(1-\vartheta) \mathrm{E}(\gamma(\theta) \mid \underline{\mathrm{t}})
$$

Also, by choosing $\rho(\mathrm{r}(\theta), \delta)=\mathrm{e}^{\mathrm{k}(\delta-\gamma(\theta))}-\mathrm{k}(\delta-\gamma(\theta))-1 \quad$ and $\mathrm{q}(\theta)=1 \quad$ in (13) we get as the balanced

LINEX loss function written as

$$
\mathrm{L}_{\lambda, \xi}^{*}(\gamma(\theta), \delta)=\vartheta\left(\mathrm{e}^{\mathrm{k}(\delta-\xi)}-\mathrm{k}(\delta-\xi)-1\right)+(1-\vartheta)\left(\mathrm{e}^{\mathrm{k}(\delta-\gamma(\theta))}-\mathrm{k}(\delta-\gamma(\theta))-1\right)
$$

For which the Bayes estimate of $\gamma(\theta)$ is based on the loss function of (16) takes the form

$$
\delta_{\omega, \xi}^{*}(\underline{\mathrm{t}})=\frac{-1}{\alpha} \ln \left[\vartheta \mathrm{e}^{-\alpha \hat{\xi}(\underline{\mathrm{t}})}+(1-\vartheta) E\left[\mathrm{e}^{-\alpha \gamma(\theta)}|\underline{\mathrm{t}}|\right]\right]
$$

Suppose that the prior knowledge about the parameters $\alpha$ and $\theta$ are assumed to be unknown, Nassar and Eissa (2004) and Kim et al. (2011), suggested a bivariate prior density is given by:

$$
\pi(\alpha, \theta)=\pi_{1}(\theta \mid \alpha) \pi_{2}(\alpha),
$$

where $\pi_{1}(\theta \mid \alpha)$ is a gamma prior density function when $\alpha$ is known

$$
\pi_{1}(\theta \mid \alpha)=\frac{\alpha^{-v}}{\Gamma(v)} \theta^{v-1} \mathrm{e}^{\frac{-\theta}{\alpha}} \quad ; \theta>0, \alpha \& v>0
$$

and $\pi_{2}(\alpha)$ is a gamma prior density function

$$
\pi_{2}(\alpha)=\frac{b^{-\tau}}{\Gamma(\tau)} \alpha^{\tau-1} e^{\frac{-\alpha}{b}} \quad ; \alpha>0, \tau \& b>0
$$


Is an exponential density function. Here, $v, \alpha$ and $\mathrm{b}$ are assumed to be known. Therefore, the bivariate prior density function of $\alpha$ and $\theta$ can be written as:

$$
\pi(\alpha, \theta)=\frac{1}{b \Gamma(v+\tau)} \alpha^{\tau-v-1} \theta^{v-1} \mathrm{e}^{-\left(\alpha^{2}+\mathrm{b} \theta\right) / \mathrm{b} \alpha}, \quad \alpha, \theta>0
$$

The joint posterior probability density function of $\alpha$ and $\theta$ is obtained from (17) and (18) and written as:

$$
\pi^{*}(\alpha, \theta \mid \mathrm{t})=\mathrm{T}^{-1} \alpha^{\mathrm{n}+\tau-v-1} \theta^{\mathrm{n}+v-1} \mathrm{e}^{-\left(\alpha^{2}+\mathrm{b} \theta\right) / \mathrm{b} \alpha} \varphi(\alpha, \theta)
$$

where

$$
\mathrm{T}=\int_{0}^{\infty} \int_{0}^{\infty} \alpha^{\mathrm{n}+\tau-v-1} \theta^{\mathrm{n}+\nu-1} \mathrm{e}^{-\left(\alpha^{2}+\mathrm{b} \theta\right) / \mathrm{b} \alpha} \varphi(\alpha, \theta) \mathrm{d} \alpha \mathrm{d} \theta
$$

and

$$
\varphi(\alpha, \theta)=\prod_{j=1}^{m} \psi_{j} \eta_{j}^{\theta} \prod_{j=1}^{m-1} \eta_{j}^{n} \theta \eta_{n}^{\theta(k-1)}
$$

Under the SEL function, and by using (15) the Bayes estimate of $\mathrm{k}$ function $\lambda=\lambda(\alpha, \theta), \theta$ is given by

$$
\hat{\lambda}_{\mathrm{BS}}=\mathrm{E}(\lambda \mid \underline{\mathrm{t}})=\frac{\int_{\alpha} \int_{\theta} \lambda(\alpha, \theta) \mathrm{L}(\alpha, \theta \mid \underline{\mathrm{t}}) \pi(\alpha, \theta) \mathrm{d} \alpha \mathrm{d} \theta}{\int_{\alpha} \int_{\theta} \mathrm{L}(\alpha, \theta \mid \underline{\mathrm{t}}) \pi(\alpha, \theta) \mathrm{d} \alpha \mathrm{d} \theta}
$$

or

$$
\hat{\lambda}_{\mathrm{BS}}=\vartheta \hat{\lambda}_{\mathrm{BS}}+(1-\vartheta) \mathrm{T}^{-1} \int_{0}^{\infty} \int_{0}^{\infty} \alpha^{\mathrm{n}+\tau-v-1} \theta^{\mathrm{n}+v-1} \mathrm{e}^{-\left(\alpha^{2}+\mathrm{b} \theta\right) / \mathrm{b} \alpha} \varphi(\alpha, \theta) \mathrm{d} \alpha \mathrm{d} \theta
$$

Also, based on the LINEX loss function, the Bayes estimate the function $\lambda$ is obtained by using (16) and written as

$$
\hat{\lambda}_{\mathrm{BS}}=\frac{-1}{\alpha} \ln \left[\vartheta \mathrm{e}^{-\alpha \widehat{\lambda}_{\mathrm{ML}}+(1-\vartheta) \mathrm{k}^{-1}} \int_{0}^{\infty} \int_{0}^{\infty} \alpha^{\mathrm{n}+\tau-v-1} \theta^{\mathrm{n}+\nu-1} \mathrm{e}^{-\left(\alpha^{2}+\mathrm{b} \theta\right) / \mathrm{b} \alpha} \varphi(\alpha, \theta) \mathrm{d} \alpha \mathrm{d} \theta\right]
$$

where $\hat{\lambda}_{\mathrm{ML}}$ is the ML estimate of $\lambda$ and $\varphi(\alpha, \theta)$ is as given by (23).

It may be noted, from (24) and (25), that the Bayes estimates of the function $\varphi=\varphi(\alpha, \theta)$ contains double integrals that cann't be obtained in simple closed form and numerical techniques must be used for computations. It used Nassar and Eisa (2004) in the form:

$$
\begin{array}{rlrl}
\text { If } \mathrm{g}(\alpha, \theta) & =\alpha \text {. Then } & \widehat{\alpha}_{\mathrm{s}}=\alpha+\psi_{1} \\
\text { If } \mathrm{g}(\alpha, \theta) & =\theta \text {. Then } & \widehat{\theta}_{\mathrm{S}}=\theta+\psi_{2} \\
\text { If } \mathrm{g}(\alpha, \theta)=\mathrm{e}^{-\mathrm{c} \alpha} \text {. Then } & \widehat{\alpha}_{\mathrm{L}}=\alpha-\frac{1}{\mathrm{c}} \ln \left(1-\mathrm{c} \psi_{1}+\frac{\mathrm{c}^{2} \mathrm{H}}{\mathrm{c}^{2 N}}\right), \mathrm{c} \neq 0 \\
\text { If } \mathrm{g}(\alpha, \theta)=\mathrm{e}^{-\mathrm{c} \theta} \text {. Then } & \widehat{\theta}_{\mathrm{L}}=\theta-\frac{1}{\mathrm{c}} \ln \left(1-\mathrm{c} \psi_{2}+\frac{\mathrm{c}^{\mathrm{G}}}{2 \mathrm{~N}}\right), \mathrm{c} \neq 0
\end{array}
$$

\section{Simulation Study and Comparisons}

The purpose of this section is to compare the performance of the different methods of estimation discussed in the previous sections. We analyze a real data set for illustrative purpose is to be analyzed. A simulation study is also carried out to check the behavior of the proposed methods and to assess the statistical performances of these estimates, a simulation study is conducted. The root mean square errors (RMSEs) using generated random samples of different sizes are computed for each estimator.

Applying the algorithm of Balakrishnan and Sandhu (1995), we used the following steps to generate a progressive Type II censored sample from the exponentiated Weibull distribution.

The random samples are generated as follows:

1. Generating $\mathrm{m}$ independent $\mathrm{U}(0,1)$ random variables $\left(\mathrm{Z}_{\mathrm{i}}\right)$ that follow the regular distribution within the period $(0,1)$.

$$
\mathrm{U}_{\mathrm{i}} \sim \mathrm{U}(0,1), \mathrm{i}=1,2, \ldots, \mathrm{n}
$$

where $U_{i}$ is a continuous random variable.

2. The values given to the progressive monitoring system are set in the following method. 


$$
Y_{i}=Z_{i}^{1 /\left(i+R_{n}+R_{n-1}+\ldots+R_{n-i+1)}\right.} \quad, \quad i=1,2, \ldots, n
$$

3. From $\operatorname{step}(2)$, we set $\quad U_{i}=1-Y_{n} Y_{n-1} \ldots Y_{n-i+1} \quad, i=1,2, \ldots, n$.

Then $U_{1}, U_{2}, \ldots, U_{m}$ is a progressive Type II censored sample of size $m$ from $U(0,1)$.

4. For a given value of $b$, generate values of $\alpha$ and $\theta$ from the joint prior density given by (21).

Using the result for $\alpha$ and $\theta$ from step (4), we generated a censored sample of size m from the exponentiated Weibull distribution(EWD), using the inverse CDF.

$$
\mathrm{T}_{\mathrm{i}}=\left(-\ln \left[1-\mathrm{U}_{\mathrm{i}}^{\frac{1}{\theta}}\right]\right)^{\frac{1}{\alpha}}, \quad \mathrm{i}=1,2, \ldots, \mathrm{n}
$$

which is the required progressive Type II censored sample of size $\mathrm{m}$ from the EWD.

We obtained the MLEs, $\widehat{\alpha}_{\text {MLE }}$ and $\widehat{\theta}_{\text {MLE }}$ of parameters $\alpha$ and $\theta$, by iteratively solving Eg. (8) and(9). Substituting $\widehat{\alpha}_{\text {MLE }}$ and $\widehat{\theta}_{\text {MLE }}$ of parameters $\alpha$ and $\theta$.

Table (1). Estimated means for different estimates $\theta$ and $\alpha$ for all different values $C$

\begin{tabular}{ccccccc}
\hline $\mathrm{C}$ & $\hat{\theta}_{M}$ & $\hat{\theta}_{S}$ & $\hat{\theta}_{L}$ & $\hat{\alpha}_{M}$ & $\hat{\alpha}_{S}$ & $\hat{\alpha}_{L}$ \\
\hline-3 & 4.68741 & 4.59842 & 6.25681 & 2.6871 & 1.8547 & 2.4561 \\
-1 & & & 5.95874 & & & 2.1032 \\
0 & & & 5.76583 & & & 2.0056 \\
1 & & & 5.45982 & & & 1.9024 \\
3 & & & 4.89547 & & & 1.8245 \\
\hline
\end{tabular}

7. In order to compare the different estimators of the parameters, the assumed different sizes were identified for sample $(n=20,40,60)$ and frequency of experiment the 1000 times, and computed the means and the LMSEs for different censoring sizes $\mathrm{m}$ and censoring schemes. The samples were simulated by using the algorithm described in Balakrishnan and Sandhu (1995).

A simulation was conducted in order to study the properties and compare the performance of the Bayes estimator with maximum likelihood estimator.

The mean square error (MSE) of the Bayes estimations and maximum likelihood estimations are computed over different combination of the censored random scheme as shown in Tables (2-8). To assess the effect of the shape parameters $\alpha$ and $\theta$, from the above tables one can see that the asymmetric Bayes estimates.

Our computational results for the means and LMSE are computed in the above steps for the case of unknown $\alpha$ and $\theta$, where the values of the parameters used are $b=5$ and $v=4$ yielding $\alpha=2.09581$ and $\theta=4.69824$ (as true values). The estimations are conducted on the basis of various progressive Type II censoring schemes which include complete and Type II censoring. For given the progressive censoring scheme $\mathrm{R}=(0,1,2,1,0,1,2,1)$ and various values of $\mathrm{c}$, the MLE and Bayes estimates for the parameters $\alpha$ and $\theta$ are as in the following Table 1.To assess the effect of the shape parameters $\alpha$ and $\mathrm{b}$ from Table 1,we see that the asymmetric Bayes estimates of the parameters $\alpha$ and $\theta$ are overestimates for $\mathrm{c}<0$. Otherwise, the effect of the $\mathrm{c}$ it gives more weight to underestimation. If $\mathrm{c}$ is near to 0 , then the Bayes estimates are almost the same as the estimates under SEL. This is one of the useful properties of working with the Linex loss function.

We study how much the estimated means and variances affected by censoring schemes, which have all surviving items removed from the experiment at the first failure point $t_{1}$, midpoint and end point tm, respectively. From the results in Table 2, it is seen that the case of all items removed at the first failure time point $\mathrm{t} 1$ is better than the other case. Table 2 also provide the estimated means, their variances, covariance's and $95 \%$ approximate confidence intervals for the parameters. 
Table 2. Estimated (Means, Variance ,Covariance , and 95\% Confidence Interval (CI) for $\theta$ and $\alpha \quad(m=20, n=6-16)$

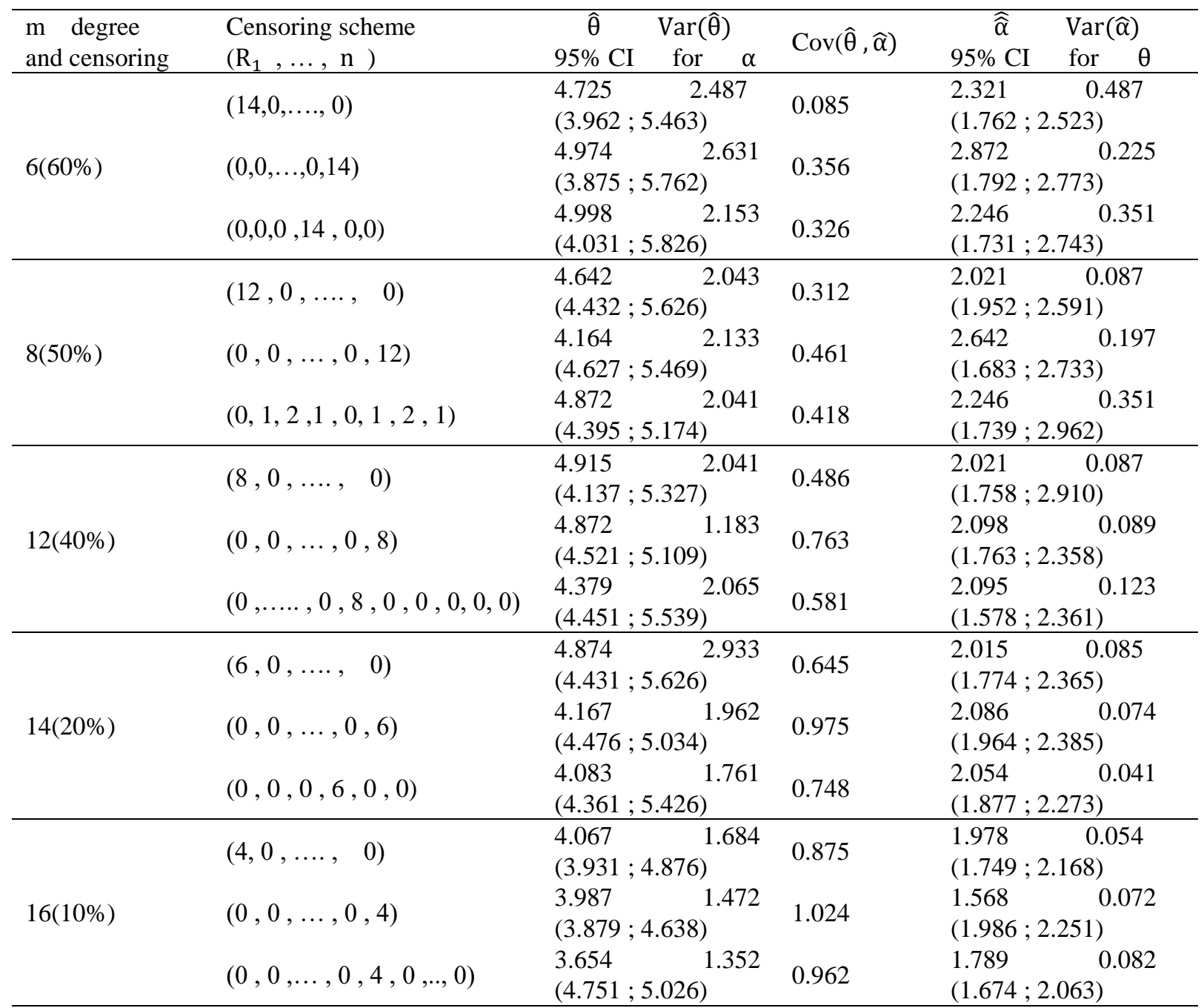

Tables 3, 4 display the estimated mean of MLE and the Bayes estimates relative to SEL and Linex loss functions of the parameters and its corresponding RMSE for different censoring schemes, respectively. Our experiments involved carrying out three cases with different censoring schemes. The first case was that all items failed, the second case is that Type II censoring, and the last case is that of progressive Type II censoring, respectively. We see that the Bayes estimates to be better than the MLE and the Bayes estimates relative to Linex loss function performed better than the others in the sense of comparing the RMSE of the estimates. This was true for both complete and Type II censored samples.

Table 3. Estimated means and RMES of various estimator $\theta$ for $\mathrm{m}=20$ and $\mathrm{C}=1$

\begin{tabular}{lccc}
\hline Censoring scheme & $\hat{\theta}_{\mathrm{M}}$ & $\hat{\theta}_{\mathrm{S}}$ & $\hat{\theta}_{\mathrm{L}}$ \\
\hline \multirow{2}{*}{ All items failed } & 4.8725 & 4.7693 & 4.6854 \\
& $(1.6587)$ & $(1.2473)$ & $(1.0942)$ \\
\hline \multirow{2}{*}{ Type II censoring } & 4.9163 & 4.8905 & 4.6792 \\
& $(1.7528)$ & $(1.5491)$ & $(0.9983)$ \\
\hline \multirow{2}{*}{$(0,1,2,1,0,1,2,1)$} & 4.8726 & 4.8094 & 4.5173 \\
$(1,1,1,1,1,1,1,1,1,1)$ & $(1.7839)$ & $(1.1684)$ & $(0.8968)$ \\
\hline \multirow{2}{*}{$(2,2,2,2,2,0,0,0,0,0)$} & 4.8719 & 4.8429 & 4.3768 \\
& $(1.5185$ & $(1.2586)$ & $(1.0986)$ \\
\hline \multirow{2}{*}{$(0,0,0,0,0,2,2,2,2,2)$} & 4.8647 & 4.8235 & 4.4783 \\
& $(1.4286)$ & $(1.3276)$ & $(0.9164)$ \\
\hline
\end{tabular}


Table 4. Estimated means and RMES of various estimator $\alpha$ for $\mathrm{m}=20$ and $\mathrm{C}=1$

\begin{tabular}{llll}
\hline Censoring scheme & $\widehat{\alpha}_{\mathrm{M}}$ & $\widehat{\alpha}_{\mathrm{S}}$ & $\widehat{\alpha}_{\mathrm{L}}$ \\
\hline \multirow{2}{*}{ All items failed } & $\begin{array}{l}1.6747 \\
(0.3558)\end{array}$ & $\begin{array}{l}1.6623 \\
(0.3079)\end{array}$ & $\begin{array}{l}1.6298 \\
(0.2962)\end{array}$ \\
\hline \multirow{2}{*}{ Type II censoring } & $\begin{array}{l}2.2174 \\
(0.7804)\end{array}$ & $\begin{array}{l}2.0975 \\
(0.67931)\end{array}$ & $\begin{array}{l}1.8952 \\
(0.6153)\end{array}$ \\
\hline \multirow{2}{*}{$(0,1,2,1,0,1,2,1)$} & $\begin{array}{l}2.1549 \\
(0.5839)\end{array}$ & $\begin{array}{l}1.9762 \\
(0.5184)\end{array}$ & $\begin{array}{l}1.8963 \\
(0.4869)\end{array}$ \\
\hline \multirow{2}{*}{$(1,1,1,1,1,1,1,1,1,1)$} & 2.0735 & 1.9583 & 1.8769 \\
& $(0.4793$ & $(0.4284)$ & $(0.3985)$ \\
\hline \multirow{2}{*}{$(2,2,2,2,2,0,0,0,0,0)$} & 2.0683 & 1.9165 & $(0.3179)$ \\
\hline \multirow{2}{*}{$(0,0,0,0,0,2,2,2,2,2)$} & $(0.3962)$ & $(0.3481)$ & 1.8492 \\
& 1.9894 & 1.8935 & $(0.3981)$ \\
\hline
\end{tabular}

Our simulation study is extended to contain different sample sizes such as $\mathrm{m}=40$ (see Tables 5,6 ) and $\mathrm{m}=60$ (see Table 7,8). These results are similar to that of sample size $m=20$. Tables 5, 6, 7 and 8 and present estimated means and RMSEs of various estimator for $\mathrm{m}=40$ and $\mathrm{m}=60$, respectively. Accordingly, as the number of each sample size increases, the variance and confidence intervals decrease. As shown by Tables 5, 6, 7 and 8, these results are alike in the case of size $\mathrm{m}=20$. In the sense of comparing the RMSE of the estimates, the Bayes estimates are better than the MLE. Even if the sample size is enlarged, expected results are the same.

Table 5. Estimated means and RMES of various estimator $\alpha$ for $\mathrm{m}=40$ and $\mathrm{C}=1$

\begin{tabular}{|c|c|c|c|}
\hline Censoring scheme & $\ddot{\theta}_{M}$ & $\tilde{\theta}_{S}$ & $\dot{\theta}_{\mathrm{L}}$ \\
\hline All items failed & $\begin{array}{l}1.9834 \\
(0.2396)\end{array}$ & $\begin{array}{l}1.8942 \\
(0.2173)\end{array}$ & $\begin{array}{l}1.8837 \\
(0.1893)\end{array}$ \\
\hline Type II censoring & $\begin{array}{l}1.9947 \\
(0.2849)\end{array}$ & $\begin{array}{l}1.9486 \\
(0.2683)\end{array}$ & $\begin{array}{l}1.9173 \\
(0.2209)\end{array}$ \\
\hline$(1,1,1,1,1,1,1,1,1,1,1,1,0,0,0,0,0,0,0,0,0,0,0,0)$ & $\begin{array}{l}1.9718 \\
(0.2792)\end{array}$ & $\begin{array}{l}1.9395 \\
(0.2594)\end{array}$ & $\begin{array}{l}1.8968 \\
(0.2465)\end{array}$ \\
\hline$(0,0,0,0,0,0,0,0,0,0,0,0,1,1,1,1,1,1,1,1,1,1,1,1)$ & $\begin{array}{l}1.9160 \\
(0.2903)\end{array}$ & $\begin{array}{l}1.8759 \\
(0.2598)\end{array}$ & $\begin{array}{l}1.8791 \\
(0.2547)\end{array}$ \\
\hline $\begin{array}{l}(2,0,0,0,2,0,0,0,2,0,0,0,2,0,0,0,2,0,0,0,2,0, \\
0,0)\end{array}$ & $\begin{array}{l}1.9751 \\
(0.2604)\end{array}$ & $\begin{array}{l}1.9356 \\
(0.2361) \\
\end{array}$ & $\begin{array}{l}1.9035 \\
(0.2109)\end{array}$ \\
\hline $\begin{array}{l}(0,0,2,0,0,0,0,4,0,0,1,0,0,0,2,0,1,0,0,1,0,2, \\
0,0)\end{array}$ & $\begin{array}{l}1.8963 \\
(0.2758) \\
\end{array}$ & $\begin{array}{l}1.8496 \\
(0.2479)\end{array}$ & $\begin{array}{l}1.8390 \\
(0.2418) \\
\end{array}$ \\
\hline
\end{tabular}

Table 6. Estimated means and RMES of various estimator $\theta$ for $\mathrm{m}=40$ and $\mathrm{C}=1$

\begin{tabular}{|c|c|c|c|}
\hline Censoring scheme & $\widehat{\alpha}_{M}$ & $\widehat{\alpha}_{\mathrm{S}}$ & $\widehat{\alpha}_{L}$ \\
\hline All items failed & $\begin{array}{l}4.6791 \\
(1.2501)\end{array}$ & $\begin{array}{l}4.7051 \\
(0.9075)\end{array}$ & $\begin{array}{l}4.6379 \\
(0.6902)\end{array}$ \\
\hline Type II censoring & $\begin{array}{l}4.8108 \\
(1.0687)\end{array}$ & $\begin{array}{l}4.7892 \\
(0.9482)\end{array}$ & $\begin{array}{l}4.5718 \\
(0.7974)\end{array}$ \\
\hline$(1,1,1,1,1,1,1,1,1,1,1,1,0,0,0,0,0,0,0,0,0,0,0,0)$ & $\begin{array}{l}4.6893 \\
(0.9531)\end{array}$ & $\begin{array}{l}4.6947 \\
(0.8952) \\
\end{array}$ & $\begin{array}{l}4.4861 \\
(0.8095) \\
\end{array}$ \\
\hline$(0,0,0,0,0,0,0,0,0,0,0,0,1,1,1,1,1,1,1,1,1,1,1,1)$ & $\begin{array}{l}4.8174 \\
(0.9602)\end{array}$ & $\begin{array}{l}4.8183 \\
(0.9037)\end{array}$ & $\begin{array}{l}4.4906 \\
(0.7628)\end{array}$ \\
\hline$(2,0,0,0,2,0,0,0,2,0 \quad, 0,0,2,0,0,0,2,0,0,0,2,0,0,0)$ & $\begin{array}{l}4.6689 \\
(0.9861)\end{array}$ & $\begin{array}{l}4.6841 \\
(0.9164)\end{array}$ & $\begin{array}{l}4.4321 \\
(0.8753)\end{array}$ \\
\hline$(0,0,2,0,0,0,0,4,0,0 \quad, 1,0,0,0,2,0,1,0,0,1,0,2,0,0)$ & $\begin{array}{l}4.8417 \\
(0.8957)\end{array}$ & $\begin{array}{l}4.8310 \\
(0.8369)\end{array}$ & $\begin{array}{l}4.5481 \\
(0.7482) \\
\end{array}$ \\
\hline
\end{tabular}

Table 7. Estimated means and RMES of various estimator $\alpha$ for $\mathrm{m}=60$ and $\mathrm{C}=1$

\begin{tabular}{|c|c|c|c|}
\hline Censoring scheme & $\tilde{\theta}_{M}$ & $\bar{\theta}_{S}$ & $\tilde{\theta}_{\mathrm{L}}$ \\
\hline All items failed & $\begin{array}{l}1.8952 \\
(0.1573)\end{array}$ & $\begin{array}{l}1.8748 \\
(0.1483)\end{array}$ & $\begin{array}{l}1.8647 \\
(0.1327)\end{array}$ \\
\hline Type II censoring & $\begin{array}{l}1.9371 \\
(0.1978)\end{array}$ & $\begin{array}{l}1.9192 \\
(0.1757)\end{array}$ & $\begin{array}{l}1.9073 \\
(0.1715)\end{array}$ \\
\hline 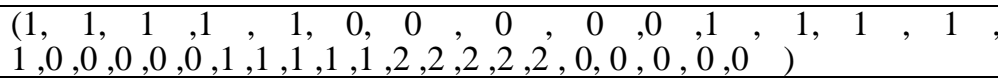 & $\begin{array}{l}1.9571 \\
(0.2194)\end{array}$ & $\begin{array}{l}1.9406 \\
(0.2082)\end{array}$ & $\begin{array}{l}1.9103 \\
(0.2009)\end{array}$ \\
\hline $\begin{array}{l}(0,0,0,0,0,1,1,1,1,1,0,0,0,0,0,1,1,1,1,1,0,0,0,0,0, \\
1,1,1,1,1,2,2,2,2,2)\end{array}$ & $\begin{array}{l}1.9641 \\
(0.2285)\end{array}$ & $\begin{array}{l}1.9372 \\
(0.2158)\end{array}$ & $\begin{array}{l}1.8987 \\
(0.2087)\end{array}$ \\
\hline $\begin{array}{l}(0,0,0,0,1,1,1,1,1,0,0,0,0,0,1,1,1,1,1,0,0,0,0,1,1,1, \\
1,1,2,2,2,2,0,0,0,0)\end{array}$ & $\begin{array}{l}1.9583 \\
(0.2194)\end{array}$ & $\begin{array}{l}1.9392 \\
(0.2061)\end{array}$ & $\begin{array}{l}1.9017 \\
(0.1983)\end{array}$ \\
\hline $\begin{array}{l}(0,2,0,0,0,4,0,0,0,1,0,0,0,0,3,0,0,0,0,0,1,1,0,0,0,4,0, \\
0,0,0,00,2,0,1,0)\end{array}$ & $\begin{array}{l}1.9582 \\
(0.2180)\end{array}$ & $\begin{array}{l}1.9468 \\
(0.2068)\end{array}$ & $\begin{array}{l}1.9072 \\
(0.2063)\end{array}$ \\
\hline
\end{tabular}


Table 8. Estimated means and RMES of various estimator $\theta$ for $\mathrm{m}=60$ and $\mathrm{C}=1$

\begin{tabular}{lllll}
\hline Censoring scheme & $\widehat{\alpha}_{\mathrm{M}}$ & $\widehat{\alpha}_{\mathrm{S}}$ & \multicolumn{1}{c}{$\widehat{\alpha}_{\mathrm{L}}$} \\
\hline All items failed & 4.7163 & 4.7591 & 4.5186 \\
& & $(0.6849)$ & $(0.6206)$ & $(0.5952)$ \\
\hline Type II censoring & & 4.6973 & 4.7268 & 4.5391 \\
& & $(0.7479)$ & $(0.7179)$ & $(0.6274)$ \\
\hline$(1,1,1,1,1,0,0,0,0,0,1,1,1,1$, & 4.6948 & 4.7149 & 4.4763 \\
$1,0,0,0,0,1,1,1,1,2,2,2,2,2,0,0,0,0,0)$ & & $(0.7408)$ & $(0.7317)$ & $(0.5962)$ \\
\hline$(0,0,0,0,0,1,1,1,1,1,0,0,0,0,0,1,1,1,1$, & 4.6742 & 4.6903 & 4.4648 \\
$1,0,0,0,0,1,1,1,1,2,2,2,2,2)$ & $(0.7286)$ & $(0.7107)$ & $(0.7013)$ \\
\hline$(0,0,0,0,1,1,1,1,1,0,0,0,0,0,1,1,1,1$, & 4.6842 & 4.7015 & 4.4596 \\
$1,0,0,0,0,1,1,1,1,2,2,2,2,0,0,0,0)$ & $(0.7359)$ & $(0.7206)$ & $(0.6387)$ \\
\hline$(0,2,0,0,0,4,0,0,0,1,0,0,0,0,3,0,0,0$, & 4.6593 & 4.6758 & 4.4432 \\
$0,0,1,0,0,0,4,0,0,0,0,0,2,0,1,0)$ & $(0.7382)$ & $(0.6851)$ & $(0.6168)$ \\
\hline
\end{tabular}

\section{Conclusions}

In this paper, we discussed the estimation problem of the unknown parameters of the exponentiated Weibull distribution based on generalized order statistics. Estimation based on progressive type-II censored samples and upper records from the exponentiated Weibull distributions are obtained as special cases. We used classical and Bayesian estimation methods to estimate the parameters. We obtained the Bayes estimates based on SE loss function using Lindley's approximation. The performance of the different estimators is compared based on simulation study in terms of their MSES. It is observed that the Bayes estimates with non-informative priors work very well in terms of MSES, also, the Bayes estimates using the informative prior have the smallest MSES among all other estimates proposed.

\section{References}

Balakrishnan, N., \& Sandhu R. A. (1995). A simple simulation algorithm for generating progressive Type II censored samples. Am Stat., 49(2), 229-230. https://doi.org/10.1080/00031305.1995.10476150

Cohen, A. C. (1963). Progressively censored samples in life testing. Technometrics 5, 327-339 Jiang R, Murthy DNP(1999) The exponentiated Weibull family: a graphical approach. IEEE Trans Reliab, 48(1), 68-72. https://doi.org/10.1080/00401706.1963.10490102

Jiang, R., \& Murthy, D. N. P. (1999). The exponentiated Weibull family: a graphical approach. IEEE Trans Reliab, 48(1), 68-72. https://doi.org/10.1109/24.765929

Kim, C., Jung, J., \& Chung, Y. (2011). Bayesian estimation for the exponentiated Weibull model under Type II progressive. censoring. Stat Papers, 52(1), 53-70. https://doi.org/10.1007/s00362-009-0203-2

Mann, N. R. (1971). Best linear invariant estimation forWeibull parameter under progressive censoring. Technometrics 13, 521-534. https://doi.org/10.1080/00401706.1971.10488815

Mudholkar, G. S., \& Hutson, A. D. (1996). The exponentiated Weibull family: some properties and a flood data application. Commune Stat Theory Methods., 25(12), 3059-3083. https://doi.org/10.1080/03610929608831886

Nassar, M. M., \& Eissa, F. H. (2003). On the exponentiated Weibull distribution. Commun. Statist. Theory Methods., 32(7), 1317-1336. https://doi.org/10.1081/STA-120021561

Nassar, M. M., \& Eissa, F. H. (2004). Bayesian estimation for the exponentiated Weibull model. Commun, Stat Theory Methods, 33(10), 2343-2362. https://doi.org/10.1081/STA-200031447

Pandey, B. N., \& Rai, O. (1992). Bayesian estimation of mean and square of mean of normal distribution using LINEX loss function. Commun.Statist. Theory Meth., 21, 3369-3391. https://doi.org/10.1080/03610929208830985

Singh, U., Gupta, P. K., \& Upadhyay, S. (2005a). Estimation of three-parameter exponentiated Weibull distribution under Type-II censoring. Journal of Statistical Planning and Inference, 134, 350-372. https://doi.org/10.1016/j.jspi.2004.04.018

Singh, U., Gupta, P. K., \& Upadhyay, S. (2005b). Estimation of parameters for exponentiated- Weibull family under Type-II censoring scheme. Computational Statistics and Data Analysis, 48, 509-523. 
https://doi.org/10.1016/j.csda.2004.02.009

Soliman, A. A. (2000). Comparison of LINEX and quadratic Bayes estimators for the Rayleigh distribution. Commun. Statist. Theory Meth., 29(1), 95-107. https://doi.org/10.1080/03610920008832471

Soliman, A. A. (2001). LINEX and quadratic approximate Bayes estimators applied to the Pareto model. Commun. Statist. Simulat. Comput., 30(1), 63-77. https://doi.org/10.1081/SAC-100001857

Viveros, R., \& Balakrishnan, N. (1994). Interval estimation of life characteristics from progressively. Censored data. Technometric, 36, 84-91. https://doi.org/10.1080/00401706.1994.10485403

Zellner, A. (1986). Bayesian estimation and prediction using asymmetric loss functions. JASA, 81, $446-451$. https://doi.org/10.1080/01621459.1986.10478289

\section{Copyrights}

Copyright for this article is retained by the author(s), with first publication rights granted to the journal.

This is an open-access article distributed under the terms and conditions of the Creative Commons Attribution license (http://creativecommons.org/licenses/by/4.0/). 